\title{
Integrationsphase: Zeit, in der das Ganze wiederhergestellt wird
}

\author{
Nadine Wickert $\cdot$ Christian Stadler
}

Online publiziert: 2. August 2018

(C) Springer Fachmedien Wiesbaden GmbH, ein Teil von Springer Nature 2018

Ausgelöst durch die EU-Flüchtlingskrise 2015 ist das Thema „Integration“ seit etlichen Monaten in aller Munde. Viele Jahre wurde Integration auf gesellschaftlicher und politischer Ebene nur nachlässig behandelt, nun aber ist das Thema zum Politikum geworden und wird im Rahmen der gesellschaftlichen Debatte um Einwanderungs- und Asylpolitik mit besonderer Brisanz diskutiert. Viel ist die Rede von Fehlern und (mitunter schwerwiegenden) Versäumnissen, und zum Teil entsteht der Eindruck, als suchten PolitikerInnen und Parteien händeringend und mehr oder weniger kopflos nach funktionierenden Konzepten von Integration.

Seinen Bedeutungsursprung hat „Integration“ im lateinischen ,integratio“, was so viel bedeutet wie ,ein Ganzes wiederherstellen“. Integration kann somit auch als das Gegenteil von Ausgrenzung bezeichnet werden. In der Psychotherapie ist Integration sozusagen systemimmanent. Denn Ziel einer Psychotherapie ist immer, dass Menschen als Ganzes wieder handlungsfähig werden, und dass sie das in der Therapie Erarbeitete in ihren Alltag überführen und schließlich gut integrieren können.

Im Psychodrama ist Integration ohnehin ein fixer Bestandteil - sowohl in der Psychodrama-Psychotherapie als auch in der psychodramatischen Beratung - und findet sich in jeder einzelnen Gruppen- sowie Einzelsitzung in der gleichnamigen, eine Einheit abschließenden Integrationsphase wieder. Die Integrationsphase besteht aus Sharing, Rollenfeedback, ggbf. Identifikationsfeedback und mitunter auch einer Prozessanalyse. Gleichzeitig lässt sich die Integrationsphase (so wie natürlich auch die beiden vorangehenden psychodramatischen Phasen der Erwärmung und der Ak-

\footnotetext{
N. Wickert

Kölblgasse 24/37, 1030 Wien, Österreich

E-Mail: mail@psychotherapie-wickert.at

C. Stadler $(\square)$

Konrad-Adenauer-Str. 27, 85221 Dachau, Deutschland

E-Mail: info@psysta.de
} 
tionsphase) nicht nur auf eine einzelne Psychodrama-Sitzung, sondern auch auf den gesamten Therapie- bzw. Beratungsprozess beziehen. Die Integrationsphase kann auch als die letzte Phase des Therapie- bzw. Beratungsprozesses gesehen werden, in der es um die Beendigung des Prozesses und den Übergang ins eigenverantwortliche Leben ohne therapeutische oder beraterische Unterstützung geht. Sowohl hinsichtlich einer einzelnen Sitzung als auch hinsichtlich des gesamten Prozess geht es sowohl um die Abrundung des Gesamtprozesses, das Einsammeln und Integrieren des bislang noch nicht Gesagten. Zusätzlich kann es um die Übertragung des Erlebten in der Surplus Reality in das Alltagsleben gehen. Die neuen Handlungsund Denkmuster werden allmählich in die gewohnheitsmäßige Handlungsstruktur integriert und auch in andere Lebensbereiche übernommen.

Das Psychodrama beinhaltet zudem eine starke gesellschaftliche Perspektive (vgl. dazu auch den Sonderband 9/2017 der Zeitschrift für Psychodrama und Soziometrie „Psychodrama und gesellschaftlicher Wandel“"). PsychodramatikerInnen haben neben dem/der einzelnen KlientIn immer auch sein/ihr Umfeld und die gesellschaftliche Entwicklung im Blick. Menschen sind und haben ein soziales Atom, und beide stehen in fortwährendem, engem Austausch miteinander. Insofern schließt die psychodramatische Sichtweise auf das Thema Integration das Individuum als auch dessen Umfeld und die gesellschaftliche Entwicklung mit ein.

So ist es auch Ziel des vorliegenden Hefts der Zeitschrift für Psychodrama und Soziometrie eine Vielzahl der verschiedenen Aspekte und Ebenen von Integration zu beleuchten. Den Anfang macht Michael Schacht mit einem Beitrag, der genau diesen Bogen spannt und sich dem Thema der Integration aber auch Desintegration sowohl auf individueller als auch auf gesellschaftlicher Ebene widmet. Schlaglichtartig beleuchtet er, was ein einzelner Mensch wie auch die Gesellschaft benötigen, damit Integration gelingen kann.

Anschließend folgen drei methodenspezifische Artikel, die die einzelnen Bestandteile der psychodramatischen Integrationsphase im Detail untersuchen: Christiane Schlüter beschäftigt sich mit dem Sharing, Reinhard Krüger mit dem Rollenfeedback und Zsusza Marlok und Christian Stadler mit dem Identifikationsfeedback. Alle drei Beiträge ermöglichen auch einen Brückenschlag von der Theorie in die Praxis und zeigen auf, wie sich die drei Techniken der Integrationsphase gewinnbringend in der Praxis einsetzen lassen.

Kersti Wei $\beta$ beschreibt wie Integrationsprozesse in der Supervision ablaufen. Sie stellt zunächst das psychodramatisch-soziodramatische Supervisionskonzept sowie die Wirkfaktoren in der Supervision vor. Anhand von zwei Fallbeispielen zeigt sie anschließend auf wie KlientInnen mit Hilfe von Supervision neue Handlungsfähigkeit gewinnen und diese im Berufsleben integrieren können.

Um andere Formen der Integration geht es in den folgenden vier Beiträgen. Gerhard Hintenberger, Silke Birgitta Gahleitner und Sonja Pasch zeigen auf, dass die Psychotherapie und Beratung schon immer stark durch die Integration anderer Methoden geprägt waren, und dass fachliche Weiterentwicklung ohne Integration gar nicht möglich wäre. Am Beispiel der verhaltenstherapeutischen Methode der Exposition in der Behandlung von Angststörungen zeigen sie auf, wie diese Methode ins Psychodrama integriert werden kann, ohne dass dies im Kontrast zur psychodramatischen Theorie ablaufen muss. 
Karsten Krauskopf und Gülay Teke beschäftigen sich mit dem Thema der schulischen Inklusion. Sie untersuchen inklusive Prozess aus den Perspektiven aller beteiligten Personen (SchülerInnen, LehrerInnen, weitere Beteiligte aus dem professionellen Netzwerk, Eltern und Familien) und legen dar, wie Inklusion von einer psychodramatischen Betrachtungsweise profitieren kann.

Roswitha Riepl schildert den Ablauf eines Soziodramas, das für eine Radiosendung inszeniert wurde. Im Sommer 2016 fand dieses Soziodrama mit tagesaktuellem Bezug zum Thema Flucht statt. Roswitha Riepl macht durch die Beschreibung des Soziodramas spürbar, welche komplexen Gefühlslagen und Handlungsmöglichkeiten dabei auf gesellschaftlicher Ebene auftraten und noch immer auftreten und wie ein Soziodrama diese bewusst und komprimiert erlebbar machen kann.

Verena Schlichtmeiers Beitrag zum Thema psychotherapeutische Begleitung von Menschen mit Flucht- und Migrationsgeschichten fokussiert die Tätigkeit des Dolmetschens als interkulturelle Perspektive in Psychotherapie und Beratung. Die Autorin setzt sich mit der Haltung in Bezug auf Selbstreflexivität, Bewertungsabstinenz bzw. Neutralität sowie in Fragen der Positionierung und Parteilichkeit auseinander. Gehören KlientInnen und PsychotherapeutInnen unterschiedlichen natio-ethno-kultureller Kontexten an, braucht es ein spezielles Vorgehen in Psychotherapie und Beratung.

Im Anschluss an die oben genannten Themenbeiträge folgen die Beiträge des offenen Teils dieser ZPS-Ausgabe. Die Aufteilung der Artikel in Themenbeiträge und offene Beiträge wurde mit dem vorangegangen Heft „Lieben“ (Ausgabe 1/2018) begonnen. Den Anfang der offenen Beiträge machen Bärbel Kress und Eva Kern. Die Autorinnen beschreiben, wie mithilfe des Psychodramas Change Management Prozesse sinnvoll erwärmt und gestaltet werden können. Veränderungen in Organisationen weisen Phasen (Change Kurve) auf und zeigen auch Strukturen (Change Pyramide); diese können anhand des Modells des kreativen Zirkels verstanden und unterstützt werden.

Im Beitrag von Christoph Zauner geht es um die Intuition und das intuitive Handeln von Psychotherapeutinnen und Psychotherapeuten. Zauner stellt vier verschiedene Ebenen von Intuition vor und erläutert wie diese in der Psychotherapie auftauchen können. Dabei macht er auch deutlich, welchen Stellenwert Intuition im Psychodrama hat.

Benjamin Aas, Günter Schiepek, Barbara Stöger, Wolfgang Aichhorn und Hannes Goditsch setzen ihren Forschungsbeitrag zur Psychodramagruppentherapie fort, der im letzten Heft der ZPS (Goditsch et al. 2018) abgedruckt war, in dem sie Anwendung, Forschung und Validierung eines Psychodramafragebogens bei PatientInnen mit ausgeprägten strukturellen Störungen im stationären Setting in den Blick nehmen. Im Mittelpunkt stehen dabei besonders die Anfangs- und die Endsituation. Im empirischen Teil des Artikels steht die Validierung eines eigens für das vorliegende Psychodrama-Konzept entworfenen Fragebogens. Ausgehend von Yaloms gruppentherapeutischen Wirkfaktoren zeigt ein 21 Item starker Fragebogen eine viergliedrige Faktorenstruktur (Neues Lernen, Interaktion, Emotion und Negativität).

Nikolaos Takis liefert den englischsprachigen Beitrag zu dieser Ausgabe. Psychodrama und Mythologie im Dienste der Suchtbehandlung lautet der Titel seiner Fallstudie. Dieser Artikel beschreibt den Einsatz griechischer Mythen in der psycho- 
dramatherapeutischen Suchtbehandlung. Es handelt sich um eine Einzelfallstudie, welche im Rahmen einer Psychodrama-Gruppe innerhalb einer Suchtentgiftungsstation vom Autor geleitet wurde. In seiner Reflexion verbindet Takis die Konzepte der psychodramatischen Rollentheorie mit der psychodynamischen Perspektive.

Ferdinand Buer erinnert uns daran, dass mit dem Psychodrama Morenos das Lachen in die Psychiatrie und Psychotherapie kam. Buer fragt, was es mit dem Lachen auf sich hat, und welche Rolle es im psychodramatischen Prozess spielt und spielen kann. Er plädiert dafür, dass PsychodramatikerInnen HumoristInnen sein sollen, denn die subversive Kraft des Humors kann bei der dringend notwendigen Entschleunigung sozialer Prozesse helfen.

Anschaulich und anhand von Fallbeispielen beschreibt Sabine Wigard wie Psychodrama im Deutschunterricht in Gymnasien und Realschulen eingesetzt werden kann, um literarische Texte zu interpretieren. Dank des Psychodramas können SchülerInnen Texte nicht nur verbal besprechen und interpretieren, sondern auch handelnd erleben. Dazu werden die Texte auf die Bühne gebracht und die SchülerInnen schlüpfen in die verschiedenen Rollen eines Textes. Auch hier hat die Integrationsphase eine wesentliche Bedeutung, da die SchülerInnen eine Verbindung zwischen Text und ihrer eigenen Lebenswelt herstellen können.

Ganz neu ist in diesem Heft die Artikel-Kategorie Toolbox. Mit jedem Beitrag der Toolbox sollen Leserinnen und Leser eine konkrete Anleitung für ein PsychodramaTool bekommen. Dies können neu entwickelte Techniken sein oder aber Variationen einer bekannten Technik, ein Arrangement oder eine Idee für die Bühnengestaltung. Den Auftakt dieser neuen Kategorie macht Bernd Kühbauer, der körperliche Erwärmungsübungen vorstellt und aufzeigt wie Körperübungen positiv auf die Begegnungsfähigkeit von KlientInnen wirken können.

In der Kategorie Forum finden sich ein Nachruf auf Andreas Ploeger, einen der ersten deutschen Psychodramatiker, sowie ein Vernetzungsbeitrag über das Psychodrama in Ungarn, ein Beitrag über das Gutachten des Wissenschaftlichen Beirats Psychotherapie (WBP), die Humanistische Psychotherapie in Deutschland nicht als wissenschaftliche Methode anzuerkennen sowie ein Beitrag über das Psychodramatische Trauma-Stabilisierungs-Programm (ETBS) für Flüchtlingsfamilien.

Sie sehen, liebe Leserin und lieber Leser, das Heft bietet dank der vielfältigen Arbeit unserer AutorInnen jede Menge Themen und Gelegenheit, Neues aufzunehmen und hoffentlich gewinnbringend in die Praxis zu integrieren. Wir wünschen Ihnen viel Freude bei der Lektüre und hoffen, dass wir Sie mit den folgenden Beiträgen der Zeitschrift für Psychodrama und Soziometrie für einen guten Start in eine neue Integrationsphase erwärmen können!

Nadine Wickert und Christian Stadler 